\title{
ensayos de cocinas españolas
}

\author{
JOSE LAORDEN \\ * Ingeniera de Caminos \\ FELIPE GOMEZ \\ Aparejador \\ Division de Instaiactones del finstituto \\ Tácnico de la Construecion y del Cemento
}

$333-1$

$S$ I N O P S IS

\begin{abstract}
Rolacion de los ensayos reellxados a postelón dol Institute Naclonel do in Viviende, tobre fince cochas do divorses fobricantes espetioles. De los onseryos se deduce que ol ealor aprovechedo para calentar al agua de los eacharros colacedas $30 \%$ dal calor comfentido en el carbon qua se quema,
\end{abstract}

\section{Introdueción}

El Ministerio de Trabajo dictó en 31 de Diciembre de 1956 una resolución convocando concurso entre fabricantes de cocinas para que presentasen modelos de gran rendimiento para ser utibizados en las viviendas españolas. Con motivo de este concurso se realizaron en el I.T.C.C. una serie de ensayos sobre las cocinas presentadas al concurso y sobre otras de uso normal en el mercado español. Los resultados extractodos de estos ensayos se describen en este artículo.

Conviene destacar la importancia económica que representa la mejora del rendimiento de una cocina y que justifica holgadamente una diferencia de precio entre modelos con buen o mal rendimiento. Una buena cocino en la que se aprovecha el $30 \%$ del calor contenido en el carbón que se quema, en vez del $20 \%$ que es la cifra de las cocinas españolas de tipo normal, puede muy bien costar 3.500 ptas. más que la cocina normal y seguir siendo un buen negocio para el usuario (1).

I11 La cocina con rendimiento del $20 \%$ puede gastar unos $2,5 \mathrm{~kg}$ de carbón mós al dia que la coclng con rentiaño o séa más de 700 ptos. de gasto extrá a nual, La co. pitolización de eslas 700 pesetos anuales, se haga como se haga, excede en mucho de las 3.500 peselas que ind. comos a rriba.

\section{Normas extranjeras de ensayos de cocinas}

Para la realización de los ensayos comparativos de las cocinos se procedió a estudiar las siguientes normas extranjeras, por carecerse de normas españolas sobre el tema:

Norma AFNOR-NF D 32-301.- - Cuisinières mètalliques d̀ feu continu pour combustibles solides. Méthode d'essaip.

Bureau de Normalisation des industries de la fonderie $(B N F)-12$, Avenue Raphaël, Paris $X V I^{*}$

Normo BRITISH STANDARD 1252: 1945.*Solid fuel cookers and combination grates».

British Standards Institution.-28 Victoria Street, London, S. W. 1.

Norma BRITISH STANDARD CODE OF PRACTICE CP 403 [1952). - Open fires heating stoves and cookers burning solid fuels.

The council for codes of practice for buitdings. Construction and engineering services. Lambeth Bridge House, London, S.E.I. 


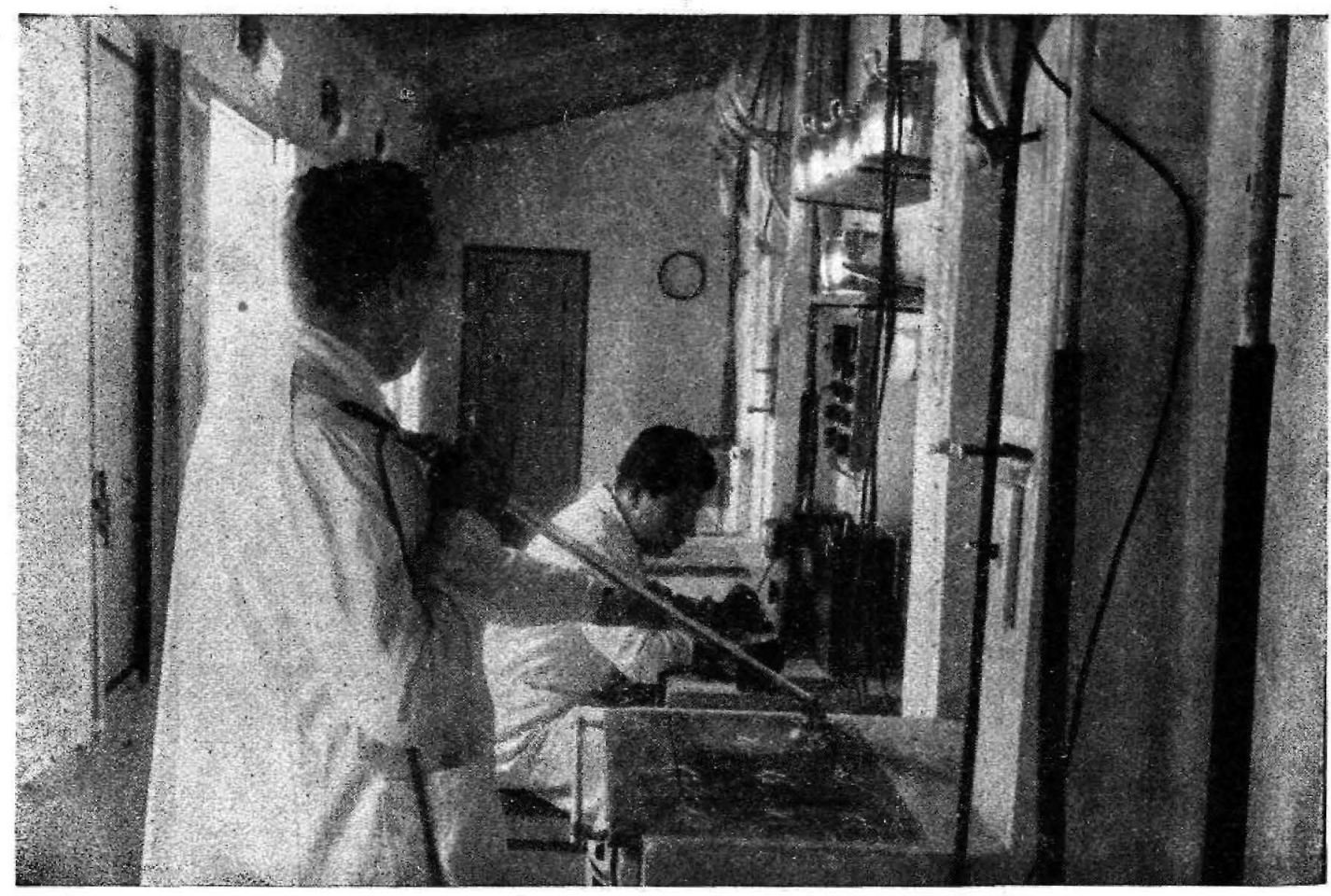

Medida de temperaturas en placas encimeras'

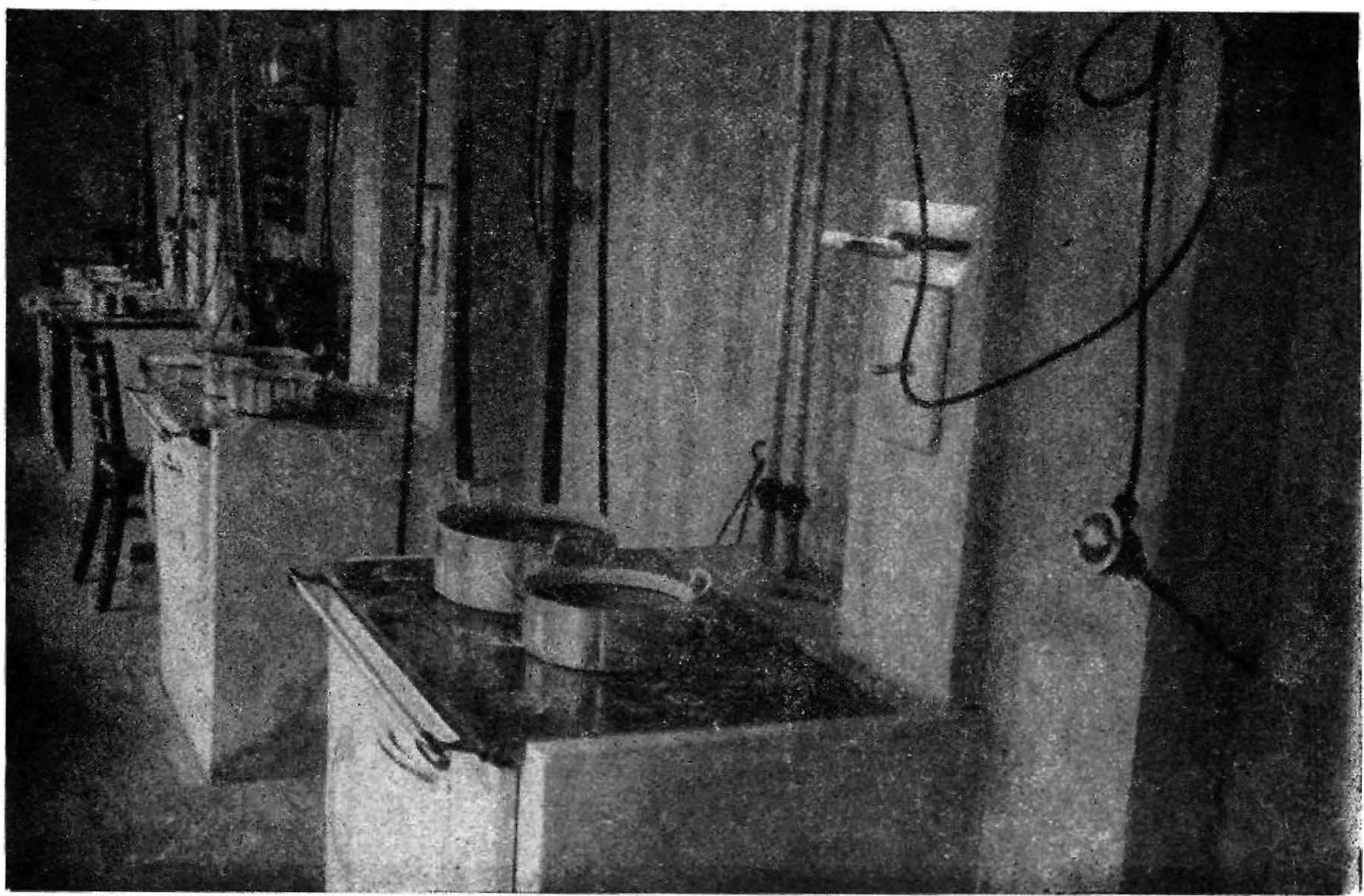

Vista general del ensayo 
Ensayo de calentamiento sobre placas encimeras.

Aparatos de medida empleados en los ensayos.
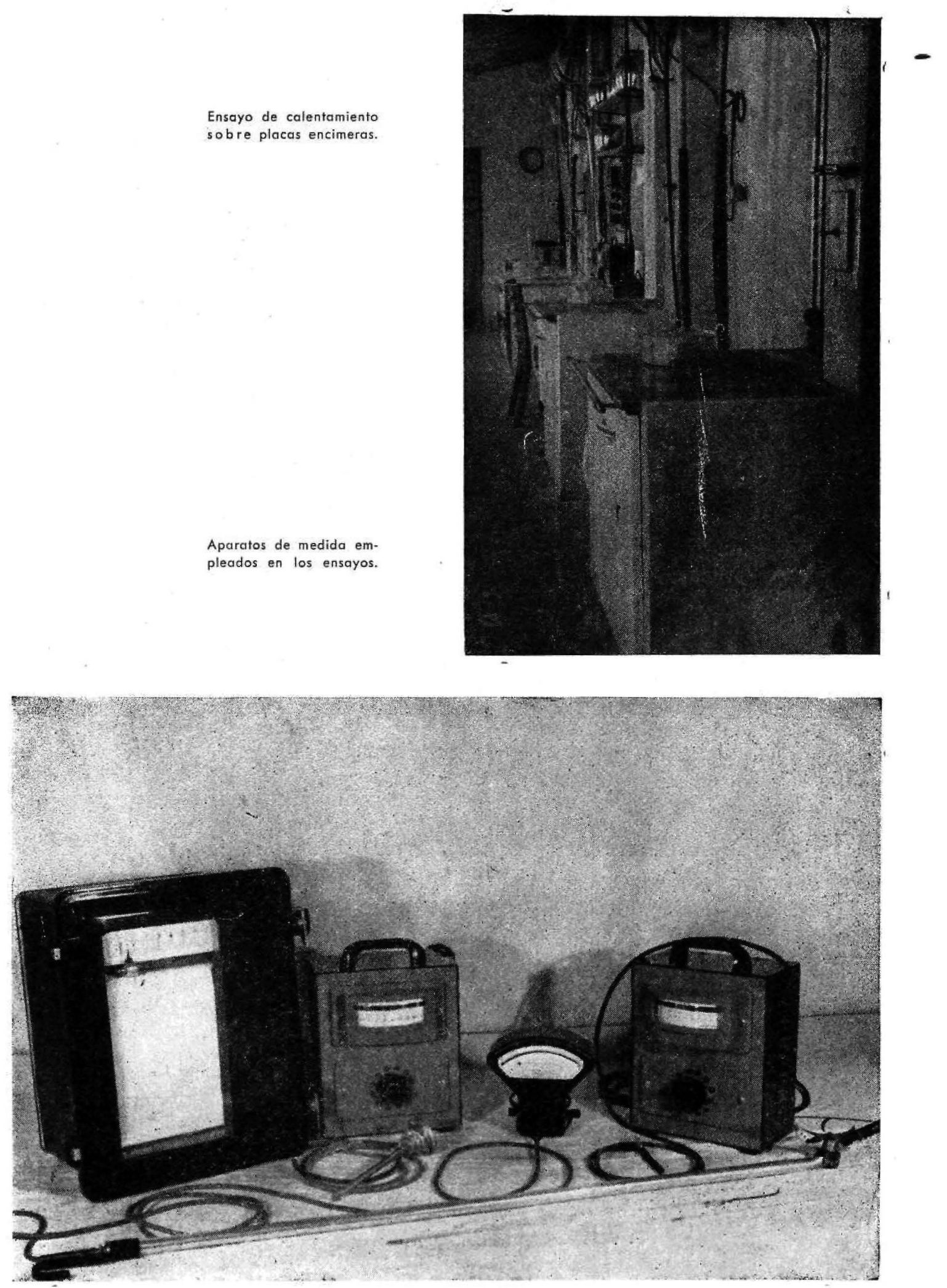

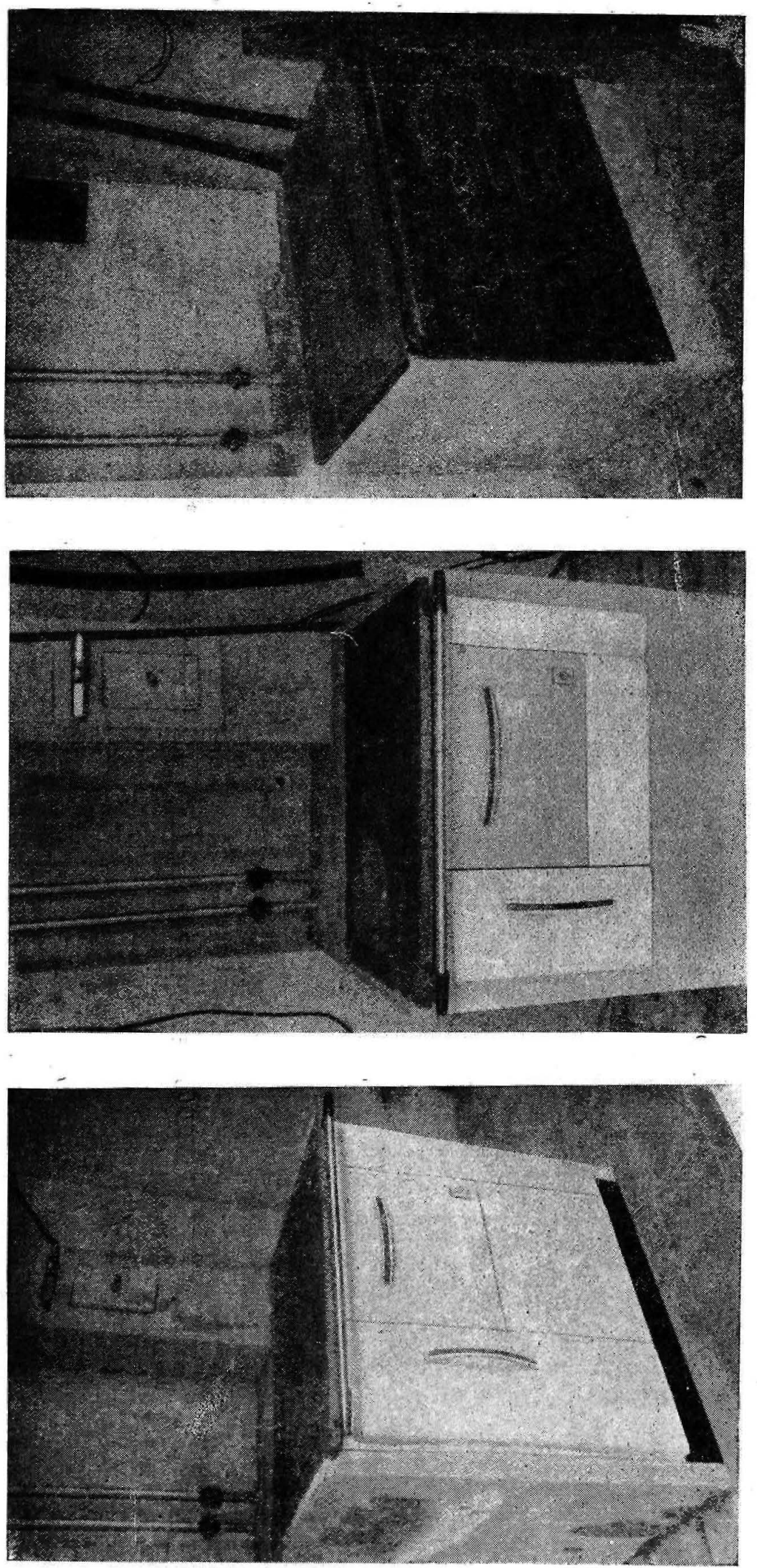

23 
Norma AMERICAN STANDARD ASA-Z21.3 - 1956/Udc 696.2: 683.9: 64.024. Approval requirements for hotel and restaurant gas ranges and unit broilers". Effective January 1, 1957 y adición a esta misma norma, con fecha de January 1 , 1958.

American gas association, Inc -420 Lexington Avenue. New York 17, New York.

Estas normas no se describen aquí, pero pueden proporcionarse por el ITCC a quienes les interesen.

\section{Finalidad de los ensayos espafioles}

Los ensayos realizados en el I.T.C.C. han sido de tipo comparativo, manteniendo simultáneamente en servicio a las cinco cocinos en un mismo local y con iguales condiciones de chimenea. (1) Con los ensayos se han querido fijar los datos siguientes:

a) Temperaturas que se producen en las placas encimeras, hornos, termos, etc.

b) Condiciones de funcionamiento de las cocinas alimentados con una cantidad igual de carbón para cada una de ellas.

c) Rendimiento de las cocinas.

d) Enscyos de fuego continuo, vuelta al régimen normal y calentamiento sobre la placa encimera según la norma francesa AFNOR - NFD 32 301 .

(1) Las condlciones de chimenea y local para la cocino número 5 differen algo de las generales, porque esla cocl-

\section{Resultedos obtenidos}

Se resumen en las tablas 1, II, III y IV que se acompañan en este artículo. Creemos que lo más importante es destacar la forma en que se obtiene el rendimiento de la cocina, con objeto de evitar confusiones con otras formas de proceder que obtienen rendimientos mucho más elevados idel orden del $65 \%$. Para nosotros el rendimiento ha sido una medida del calor aprovechado en el agua contenida en los cacharros que se calientan en la cocina y en el agua del termo. Naturalmente este rendimiento no afecto sólo a la cocina, sino al conjunto "cocina, cacharros, termo*, pero creemos que es el número de más interés para estudios de este tipo, y como los ensayos han sido simultóneos ofrece un verdadero valor de comparación real de unas cocinas con otras.

\section{Conclusiones}

Las tablas I, II, III, IV creemos que son suficientemente explícitas. Se pueden resumir diciendo que:

1. El rendimiento del conjunto kcocinacacharros-termo $*$ varía del: 21 al $30 \%$.

$2 .^{\circ}$ Los consumos de carbón de cada cocina varían de 500 a 900 gramos cada hora.

$3 .{ }^{\circ}$ Las instalaciones de ensayo del 1.T.C.C. permiten hacer un ensayo de una cocina, según las orientaciones dichas, en el plazo aproximado de unos 20 dlos, pudiendo proporcionarse los datos correspondientes a los fabricantes o a cualquier otro interesado. 
TABLA - DATOS DEL ENSAYO "O"

ENSAYO COMPARATIVO DE IAS TEMPERATURAS QUE SE PRODUCEN EN LAS PLACAS ENCIMERAS, HORNOS, TERMOS, ETC.

¿Cocinas funcionando sin batería de cocina. El agua del termo hierve a $98^{\circ} \mathrm{C}$ ]

D A $\quad$ T $O$ S

Consumo total durante los tres dias del ensayo $i k g$ de carbón] $\ldots \ldots \ldots \ldots$ Horas totales de funcionamiento durante los tres dias ..................

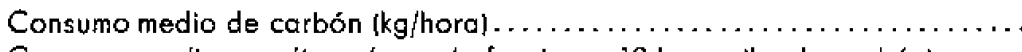
Consumo medio por dia, a base de funcionar 12 horas $(\mathrm{kg}$ de carbón) .....

Temperatura de la placa del hogar $\left(^{\circ} \mathrm{Cl}\right.$

VALORES UNA HORA DESPUES DE ENCENDIDA LA COCINA

Temperaturc en el interior del horno $1^{\circ} \mathrm{C}$

Temperatura del agua del termo $\left({ }^{\circ} \mathrm{C}\right)$

Temperatura de los humos $1^{\circ} \mathrm{C}$

Temperatura de la placa del hogar $f^{\circ} \mathrm{C}$.

VAIORES TRES HORAS DESPUES DE ENCEN DIDA LA COCINA

Temperatura en el punto eA piver figural $1^{\circ} \mathrm{Cl} \ldots \ldots \ldots$

Temperatura en el interior del horno $1^{\circ} \mathrm{Cl}$

Temperatura del agua del termo $1^{\circ} \mathrm{C}$

Temperaturo de los humos $1^{\circ} \mathrm{C}$

an.

Temperatura de la placa del hogar $1^{\circ} \mathrm{Cl}$....

VALORES SEIS HORAS DESPUES DE ENCENDIDA LA COCINA

Temperatura en el interior del horno $1^{\circ} \mathrm{C}$

Temperatura de agua del termo

${ }^{\circ} \mathrm{Cl} \ldots \ldots \ldots \ldots \ldots$

Momento en que empieza a hervir el agua del termo, horas a partir

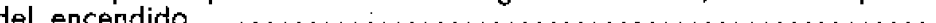

COCINA

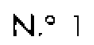

20.0

$23 \mathrm{hr} 50 \mathrm{~min}$

0,839

10,070

340 a 350

205 o 235

$355 \div 205$

72 a 84

125 a 165

320 व 370

140 a 210

110 \% 185

HIERVE

108 a 165

385 a 410

170 a 210

135 a 165

HIERVE

115 a 150

2 hr $40 \mathrm{~min}$
COCINA

N.'2

17,0

24 hr 10 min

0,703

8,450

352 , 400

162 a 285

124 a 195

52

84 a 135

231 a 355

110 a 150

95 a 155

75 a 95

$70 \circ 130$

200 a 385

155 a 220

110 a 205

50 a 92

70 a 165

$3 \mathrm{hr} 45 \mathrm{~min}$
COCINA

N. ${ }^{\circ} 3$

COCINA

N. ${ }^{\circ} 4$

COCINA

20,5

$4 \mathrm{hr} 35 \mathrm{~min}$

0,833

12,0

23 hr 5 min

0,520

6,250

$10.6 *$

: $15 \mathrm{nr} 5 \mathrm{~m} / \mathrm{n}^{*}$

0,703

250 a 410

150 a 250

138 a 190

40 o 50

105 a 150

$420 \circ 480$

68 a 110

65 a 100

38 a 56

43 a 66

335 a 450

150 a 300

$150 \propto 262$

75 a 98

110 a 190

395 a 430

65 a 95

84 a 93

60 a 69

45 a 60

430

300
$150 \circ 270$

37 a 68

418 a 435

450 y 585

210 a 300

105 a 130
95 a 130

68 a 98

HIERVE

153 a 220

$32 \propto 67$

300 a 340

130 a 170

145 a 185

39 a 53

35 a 50

260 a 370

130 a 195

125 a 223

42 a 54

40 a 50

$3 \mathrm{hr} 45 \mathrm{~min}$

$5 \mathrm{hr} 45 \mathrm{~min}$

No hierve

Para otras temperaturas en las placas encimeros, ver figuros.

En esta cocin 
TABLA II - DATOS DEL ENSAYO "b"

COCINAS FUNCIONANDO CON RECIPIENTES IGUALES, CONTENIENDO AGUA Y MANTENIENDO

SU COMBUSTION CON IGUAL CONSUMO DE CARBON

D A $\quad$ T O S

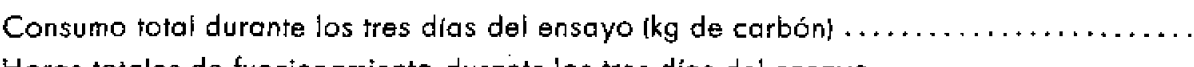

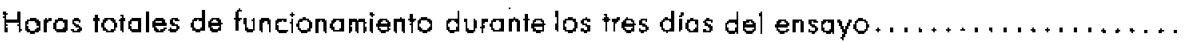

Consumo medio de carbón $\mathbf{k g} / \mathrm{hr}$. .

Consumo medio por día a base de funcionar 12 horas $(\mathrm{kg}$ de carbón) ..................

Temperatura del agua en una cazuela, sobre la placa del hogar $1^{\circ} \mathrm{C}$ Temperatura del agua en una cazuelc, al lado del hogar $l^{\circ} \mathrm{C}$......

VALORES UNA HORA DESPUES DE ENCENDIDA LA COCINA

Temperatura del agua en una olla, en el horno $1^{\circ} \mathrm{C}$.

Temperatura del agua en el termo de agua caliente $\left(^{\circ} \mathrm{C}\right) \ldots \ldots .$.

Temperatura del agua en una cozuela, sobre la placa del hogar $l^{\circ} \mathrm{C}$ Temperatura del agua en una cozvela, ol lado del hogar $1^{\circ} \mathrm{Cl} \ldots$.

VALORES TRES HORAS

QESPUES DE ENCEND

DA IA COCINA

Temperatura del agua en una olla, en el horno $l^{\circ} \mathrm{C}$

Temperatura del agua en el termo de agua caliente $\left.\right|^{\circ} \mathrm{C} \mid \ldots \ldots \ldots$

Momento en que empieza a hervir el agua en la cazuela sobre el hogar. Horas a partir

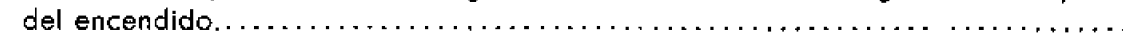
Momento en que empieza a hervir el agua de la cazuela al lado del hogar. Horas a partir del encendido. ..................................................

Momento en que llega a $90^{\circ} \mathrm{C}$ el agua del termo. Horaș a partir del encendido ......... Temperatura del agua del termo tres horas después de encendida ta cocina ${ }^{\circ} \mathrm{Cl} \ldots \ldots$....

Temperatura del agua en la olla del homo, seis horos después de encendida la cocina ${ }^{\circ} \mathrm{C}$ )

$\begin{array}{cc}\text { COCINA } & \text { COCINA } \\ \text { N. }{ }^{\circ} 1 & \text { N. }\end{array}$

16,5

$22 \mathrm{hr} 20 \mathrm{~min}$

0,740

8,880

44

34 a 58

56 a 90

77 a 96

53 ○ 72

56 a 80

71 a 83

2,5 a $3 \mathrm{hr}$

HO H:ERYE

$3,5 \mathrm{hr}$

71 a 83

76 × 90
COCINA

$N{ }^{\circ} 3$

16,5

16,5

0,740

8,880

$22 \mathrm{hr} 20 \mathrm{~min}$

0,740

8,880

56 a 98

48 a 66

41 a 57
$45 \square 68$

59 a 60

70 a 93

55 ○ 77

61 a 71

80

75 ○ 94

55 o 71

66 a 91

44095

2,5 a $3,5 \mathrm{hr}$

$1,5 \circ 2,5 \mathrm{ht}$

1,5 a $2,5 \mathrm{hr}$

HO HEERYE

HO HERVE

$4 \mathrm{hr}$

2,5 hir

95

HO HERVE

80 o 81

81 a 93

$25 \circ 64$

56 व 97

34 a 62

38 a 70

$39 \circ 90$

$67 \circ 83$
$3,33 \mathrm{hr}$

39 a 90

51 a 77
COCINA

N. ${ }^{\circ}$

13,5

$2 \mathrm{hir} 20 \mathrm{~min}$

$0,605^{*}$

$85 \circ 97$

28 a 58

23 व 41

El consumo maximo de carbón utilizado en esla coeing no gicanzo a

consumo minimo previsto pora esto ensayo en las cocinas núms. I, 2 y 3 . 
TABLA II - DATOS DEL ENSAYO "c"

RENDIMIENTO DE CADA COCINA FUNCIONANDO CON EL CARBON NECESARIO PARA CADA

UNA Y CALENTANDO AGUA EN IGUALES RECIPIENTES

La temperatura del agua de los grifos es de $12^{\circ} \mathrm{C}$ y la ebullición del agua se produce a los $98^{\circ} \mathrm{C}$; los datos se refieren al total de los tres dias de ensayos

$$
\text { D A T O S }
$$

\begin{tabular}{|c|c|c|c|c|}
\hline COCINA & COCINA & COCINA & COCINA & COCINA \\
\hline N. ${ }^{\circ}$ & N. 2 & $N: 3$ & $N^{\circ}{ }_{4}$ & $N^{\circ} S$ \\
\hline 19,0 & 19,0 & 20,0 & 12,5 & 9,81 \\
\hline $23 \mathrm{br} 15 \mathrm{~m} / \mathrm{n}$ & $23 \mathrm{hr} 15 \mathrm{~min}$ & $23 \mathrm{kr} 15 \mathrm{mins}$ & $23 \mathrm{hr} 15$ mint & 15 hr 45 ain $* * *$ \\
\hline 0,816 & 0,816 & 0,816 & 0,540 & 0,623 \\
\hline 19,0 & 19,5 & 18,7 & 21,0 & 20,37 \\
\hline 12.160 & 12.500 & 12.000 & 13.400 & 13.000 \\
\hline 6,5 & 5,3 & 2,6 & 1,8 & 0,95 \\
\hline 90 & 91 & 97 & 94 & 906 \\
\hline 510 & 420 & 220 & 508 & 63,4 \\
\hline 2,2 & 2,8 & 2,3 & 5,3 & 2,32 \\
\hline 71 & 66 & 67 & 48 & 69,2 \\
\hline 128 & 151 & 126 & 191 & 105 \\
\hline 7,3 & 7,4 & 4,8 & 8,5 & 4,36 \\
\hline 84 & 77 & 84 & 60 & 78,4 \\
\hline$\$ 25$ & 480 & 350 & 410 & 237 \\
\hline 23.300 & 27.100 & 19.670 & 6.895 & 2.375 \\
\hline 36.623 & 40.651 & 32366 & 21.404 & 15.780 \\
\hline 144.500 & 144.500 & 152.000 & 95,000 & $53.000^{* *}$ \\
\hline 25,4 & 27,1 & 21,3 & 22,6 & 29,8 \\
\hline
\end{tabular}

Consumo tatal durante los tres dias del ensayo. $-1 \mathrm{~kg}$ de carbón]........

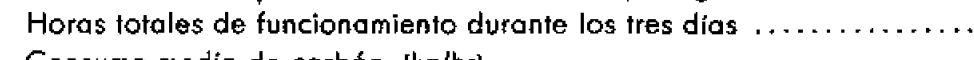

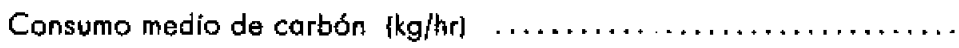

Agua evaporada en los tres recipientes.-(litrosl ..................

Calor absorbido para evaporar el agua.-|Kilocalorías\}.............

Agua que queda en la cazuela sobre el hogar*-(litros\} ...............

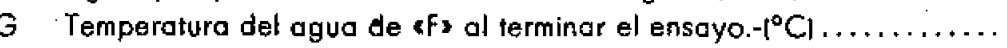

Calor absorbido en el agua que queda en (F).-[Kilocalorias)..........

Agua que queda en la cazuelo al lado del hogar". -4hitros] .............

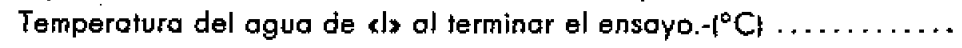

Calor absorbido en el agua que queda en th.-(Kilocalorias! ..........

L Agua que queda en la oila dei horno*.-flitrosl...................

$M$ Temperafura del agua de la olla al terminar el ensayo. $\left.-^{\circ} \mathrm{C}\right) \ldots \ldots \ldots$

$\mathrm{N}$ Calor absorbido en el agua que queda en la olla.-[Kilocalorias]........

O Calor absorbido por el termo.-1Kitocaloríasl.

......................

Calor total aprovechado por to cocina (E+H+k+N+O)--1Kilocolorias)..

Q Calor contenido en el carbón quemado $\mid$ Carbón de $7.600 \mathrm{kcal} / \mathrm{kg}$ I.-

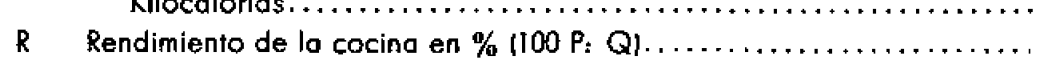

25,4

27,1

21,3

22,6

29,8

* Durante el ensayo se vo ahiodiendo agua o los recipientes, según la van evaporando.

** El corbón empleodo al ensayar esla cocina es de $5.400 \mathrm{kcal} / \mathrm{kg}$.

+ En esta cocino el ensayo ha duzado solomente dos dias y la temperatura del agua contenida en los cacharros, antes de calentarse lue de $24^{\circ} \mathrm{C}$ 


\section{TABLA IV - DATOS DEL ENSAYO"d"}

\section{ENSAYO DE FUEGO CONTINUO, DE VUELTA AL REGIMEN NORMAL Y CALENTAMIENTO SOBRE PLACA ENCIMERA}

(Estos ensayos están basados en los indicados en la norma francesa AFNOR)

\begin{tabular}{|c|c|c|c|c|c|c|}
\hline ENSAYO & DATOS & $\begin{array}{c}\text { COCINA } \\
\text { N. }\end{array}$ & $\begin{array}{c}\text { COCINA } \\
N^{\circ} 2\end{array}$ & $\begin{array}{c}\text { COCINA } \\
\text { N. } 3\end{array}$ & $\begin{array}{c}\text { COCINA } \\
N^{\circ} 4\end{array}$ & $\begin{array}{c}\text { COCINA } \\
\text { N. } 5\end{array}$ \\
\hline & $\begin{array}{l}\text { Duración del fuego continuo hasta tatal extinción .... } \\
\text { Consumo realizado por cada cocina, durante los tres } \\
\text { dias de ensayo } \mathrm{kg} / \mathrm{hr} \ldots \ldots \ldots \ldots \ldots \ldots \ldots \ldots\end{array}$ & $\begin{array}{l}17 \mathrm{hr} 45 \mathrm{~min} 0 \\
18 \mathrm{hr} \\
0,121 \text { a } 0,197\end{array}$ & $\begin{array}{l}12 \mathrm{hr} 10 \mathrm{~min} \mathrm{a} \\
18 \mathrm{hr} 50 \mathrm{~min} \\
0,196 \text { a } 0,266\end{array}$ & $\begin{array}{l}16 \mathrm{hr} 15 \min \mathrm{a} \\
19 \mathrm{hr} 5 \mathrm{~min} \\
0,134 \mathrm{a} 0,184\end{array}$ & $\begin{array}{l}6 \mathrm{hr} 45 \mathrm{~min} a \\
9 \mathrm{hr} 30 \mathrm{~min} \\
0,250 \text { a } 0,276\end{array}$ & $\begin{array}{l}22 \mathrm{hr} \\
0,136\end{array}$ \\
\hline $\begin{array}{l}\text { MARCHA EN } \\
\text { FUEGO } \\
\text { CONTINUO I" }\end{array}$ & 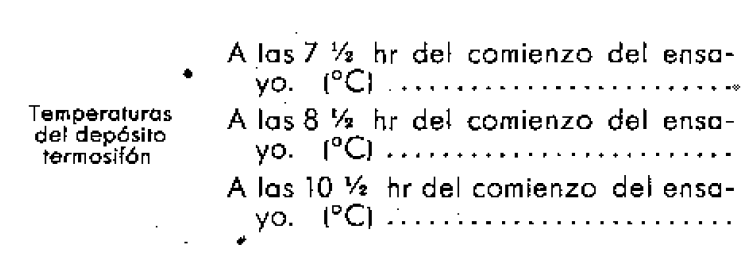 & $\begin{array}{l}61 \circ 67 \\
59 \text { a } 66 \\
53 \circ 62\end{array}$ & $\begin{array}{l}78 \circ 80 \\
77 \text { ○ } 80 \\
72 \text { ○ } 74\end{array}$ & $\begin{array}{l}54 \\
53 \\
48\end{array}$ & $\begin{array}{l}53 \\
54 \\
49\end{array}$ & $\begin{array}{l}36 \\
36,5 \\
37\end{array}$ \\
\hline $\begin{array}{l}\text { MARCHA EN } \\
\text { FUEGO } \\
\text { CONTINUOY Y } \\
\text { VUEIAAAL } \\
\text { REGMEN } \\
\text { NORMAL }\end{array}$ & $\begin{array}{l}\text { Tiempo transcurrido en volver al régimen normal a par- } \\
\text { tir de la finalización de la marcha a fuego continuo.. }\end{array}$ & $15 \min$ & $15 \mathrm{~min}$ & $15 \mathrm{~min}$ & $15 \mathrm{~min}$ & $12 \mathrm{~m} / \mathrm{n}$ \\
\hline $\begin{array}{l}\text { CALENTA- } \\
\text { MIENTOSOBRE } \\
\text { PLACA } \\
\text { ENCIMERA }\end{array}$ & 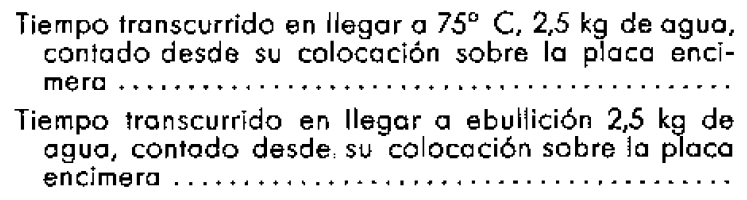 & $20 \min (*)$ & $\left.15 \min !^{*}\right)$ & $20 \min (*)$ & $\begin{array}{l}17 \min |*| \\
\left.21 \min ||^{*}\right)\end{array}$ & $\begin{array}{l}\left.8 \min l^{* * *}\right) \\
\left.12 \min l^{* * *}\right)\end{array}$ \\
\hline
\end{tabular}




\section{TEMPERATURA EN PLACAS ENCIMERAS}

\{Sin recipientes. En cada punto se ha indicado la temperatura que se produce en ${ }^{\circ} \mathrm{C}$ \} UNA HORA DESPUES DE ENCENDIDAS
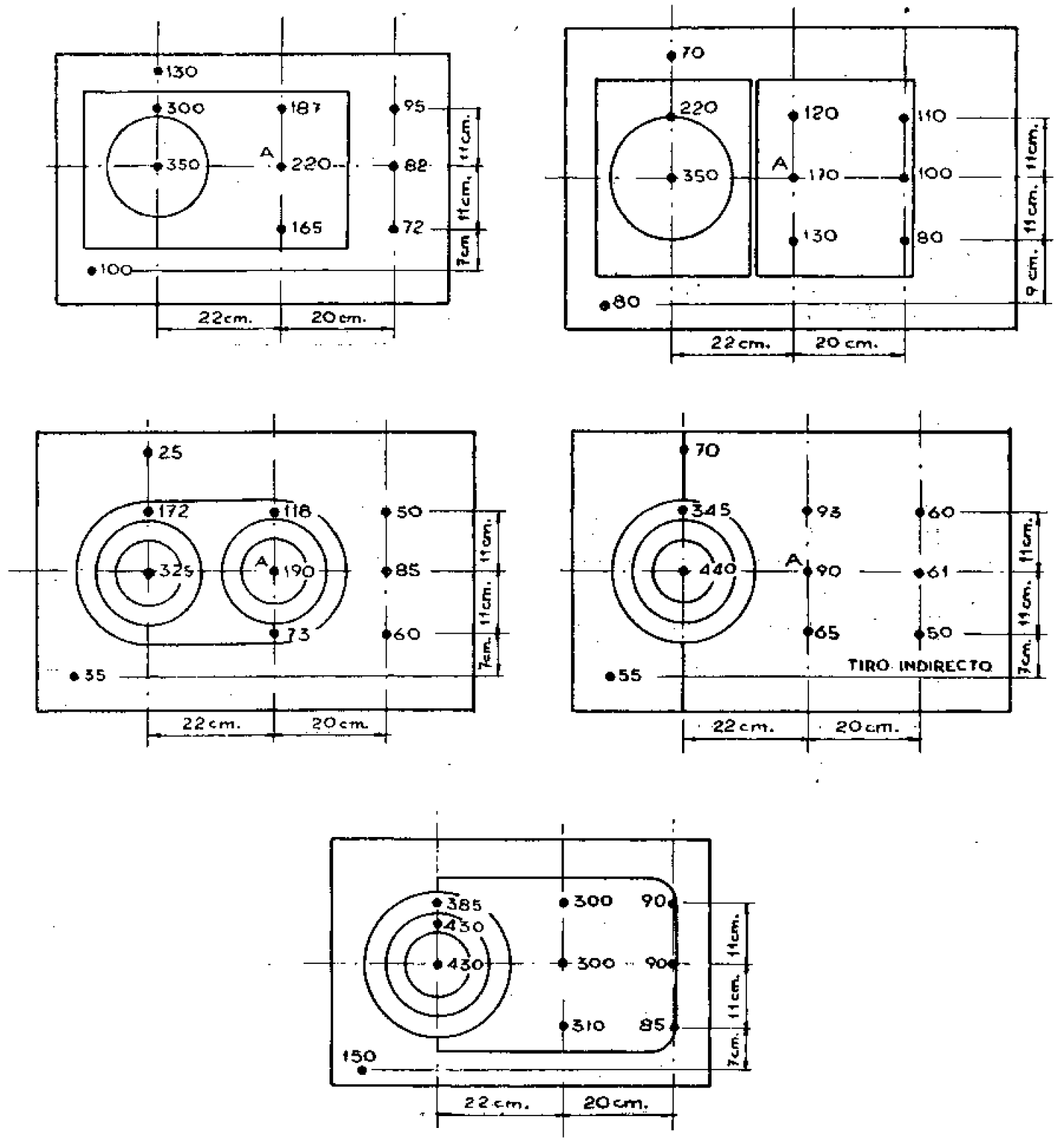


\section{TEMPERATURA EN PLACAS ENCIMERAS}

(Sin recipientes. En cada punto se ha indicado la temperatura que se produce en ${ }^{\circ} \mathrm{C}$ )

TRES HORAS DESPUES DE ENCENDIDAS
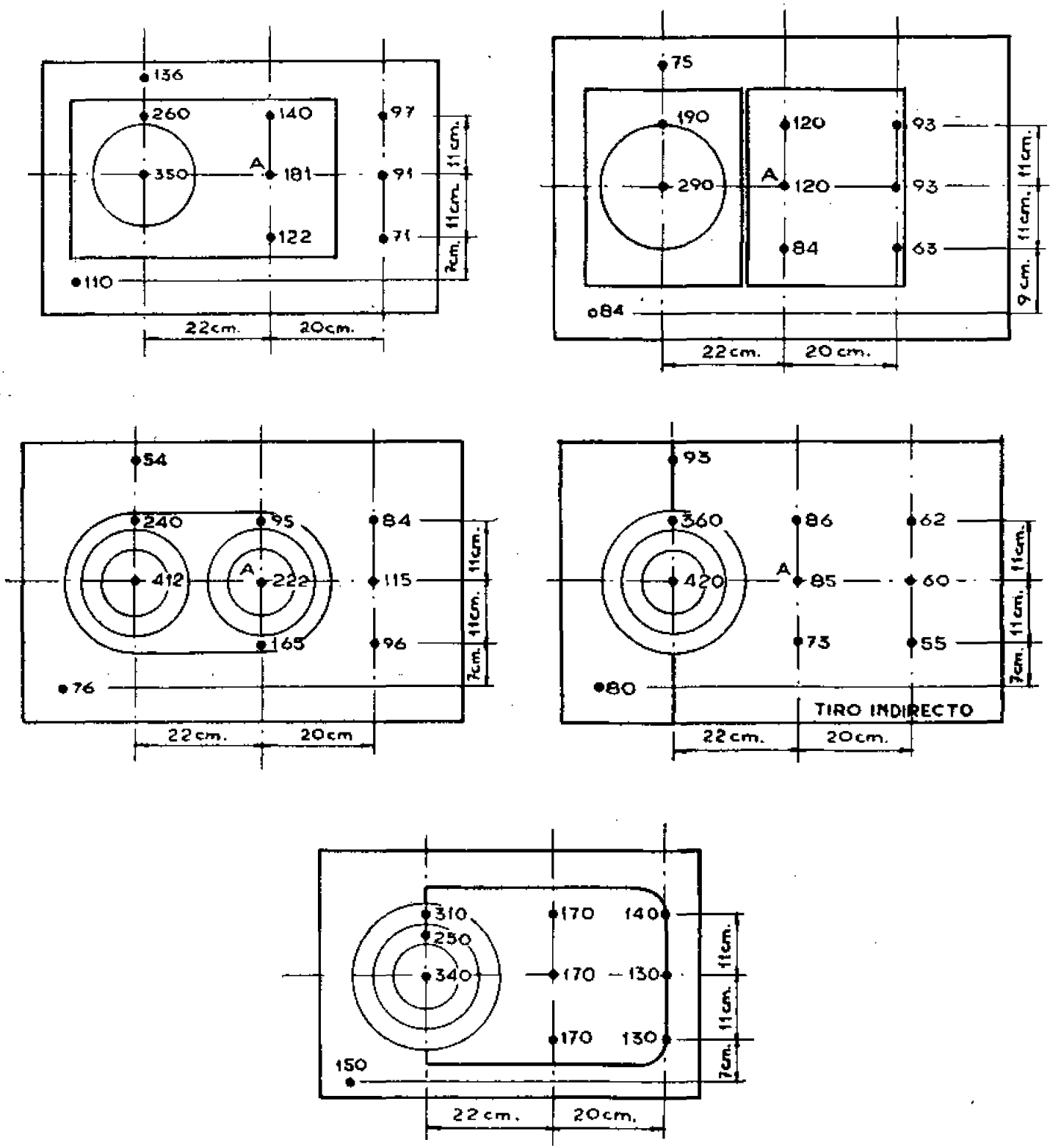


\section{TEMPERATURA EN PLACAS ENCIMERAS}

(Sin recipientes. En cada punto se ho indicado la temperatura que se produce en ${ }^{\circ} \mathrm{C}$ ) SEIS HORAS DESPUES DE ENCENDIDAS
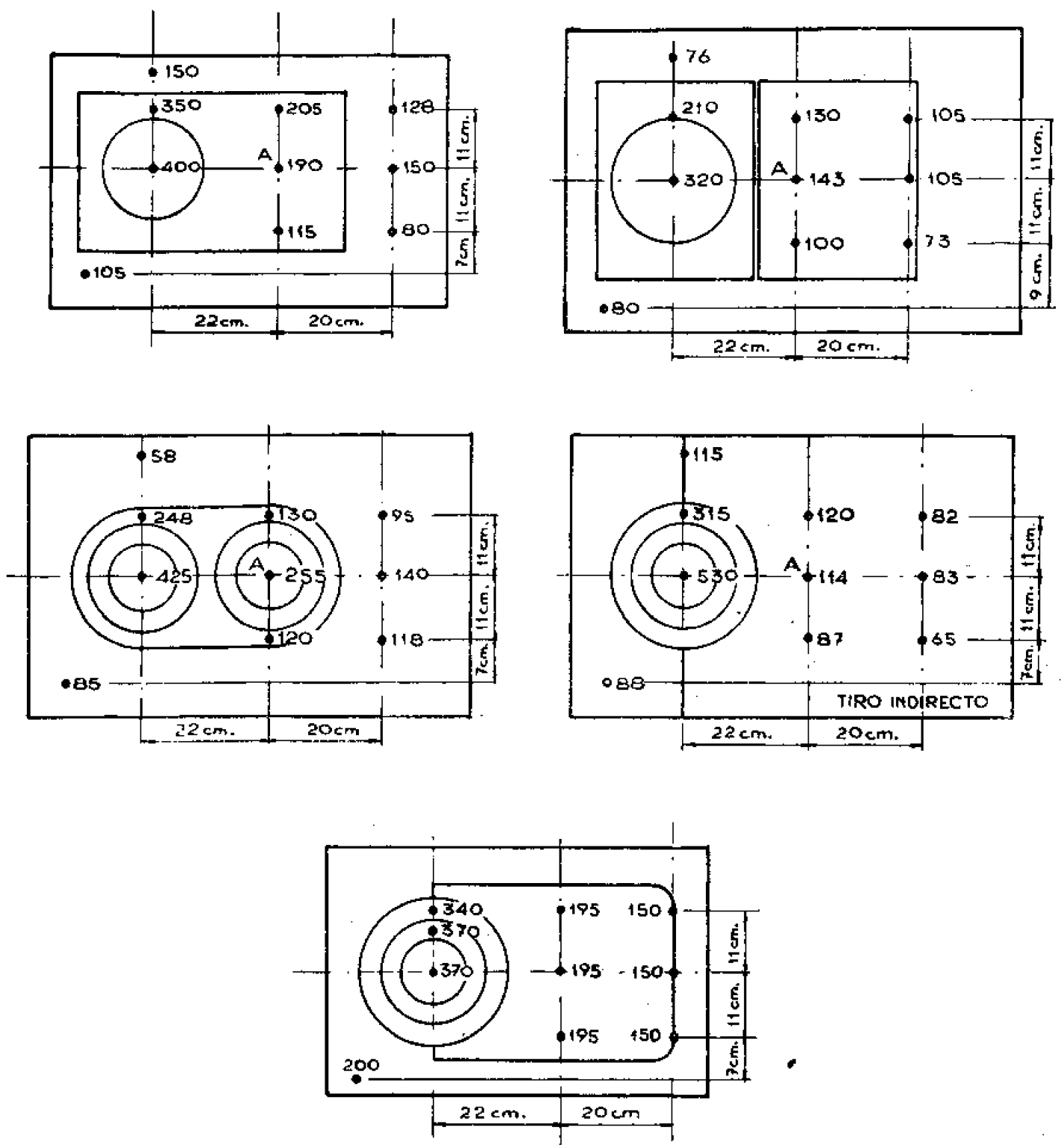